\title{
Oxygen therapy in premature low birth weight infants is associated with capillary loss and increases in blood pressure: a pilot study
}

\author{
Rajendra P. Raghuraman ${ }^{1}$ - Donovan Duffy ${ }^{2}$ Veronica A. Carroll ${ }^{1}$ Isaac Manyonda ${ }^{3}$. Tarek F. Antonios ${ }^{1,4}$
}

Received: 11 January 2019 / Revised: 28 March 2019 / Accepted: 15 April 2019 / Published online: 9 May 2019

(C) Springer Nature Limited 2019

\begin{abstract}
Low birth weight (LBW) and premature birth are known risk factors for future cardiovascular disease and in particular essential hypertension (EH). Capillary rarefaction (CR) is an established hallmark of EH and is known to occur in individuals with a history of LBW. We previously reported that LBW infants do not have CR at birth but rather increased capillary density (CD). We hypothesized that LBW infants undergo a process of accelerated CR in early life, triggered in part by oxygen therapy. We studied $26 \mathrm{LBW}$ infants, of whom 10 infants received oxygen therapy, and compared them to 14 normal birth weight (NBW) infants. We measured CD at 1, 5 and 10 days after birth and again after 40 weeks adjusted gestational age equivalent to birth at full term. We confirmed that LBW infants had higher CD at birth compared to NBW infants and found that significant structural CR occurred at term age in LBW infants who had received oxygen therapy (mean difference -22 capillaries/field, $p=0.007$ ) and in those who did not receive oxygen therapy (mean difference -29 capillaries/field, $p<0.001$ ) compared to baseline at birth. Both LBW groups showed a significant rise in BP at 40 weeks adjusted term age and the rise in systolic (mean difference $24 \mathrm{~mm} \mathrm{Hg}, p<0.0001$ ) and diastolic BP (mean difference $14 \mathrm{~mm}$ $\mathrm{Hg}, p<0.001$ ) was more pronounced in the oxygen treated group compared to the nonoxygen group (mean difference 14 $\mathrm{mm} \mathrm{Hg}, p=0.043$ and mean difference $=9 \mathrm{~mm} \mathrm{Hg} p=0.056$ respectively). In conclusion, oxygen therapy in premature LBW infants may induce significant increases in their BP in early life.
\end{abstract}

\section{Introduction}

Low birth weight (LBW $<2500 \mathrm{~g}$ ) is a recognized risk factor for the development of future essential hypertension (EH), ischemic heart disease, diabetes mellitus, obesity, and increased cardiovascular mortality in adult life $[1,2]$. The pathophysiological mechanisms are yet to be elucidated, however, it has been suggested that microcirculatory abnormalities and impaired tissue perfusion are implicated

Tarek F. Antonios

t.antonios@sgul.ac.uk

1 Molecular \& Clinical Sciences Research Institute, St. George's, University of London, London, UK

2 Neonatal Intensive Care Unit, St George's University Hospitals NHS Foundation Trust, London, UK

3 Department of Obstetrics and Gynaecology, St George's University Hospitals NHS Foundation Trust, London, UK

4 Blood Pressure Unit, St George's University Hospitals NHS Foundation Trust, London, UK in the pathogenesis of these cardiovascular disorders [3]. It is thought that the suboptimal in-utero conditions that impair the fetal growth in the first instance may affect the microvasculature and result in many structural and functional abnormalities including the reduction in spatial density of capillaries or capillary rarefaction (CR) [4]. We have previously reported that much of the $\mathrm{CR}$ in $\mathrm{EH}$ is caused by the structural absence of capillaries [5]. We have also shown significant $\mathrm{CR}$ in patients with borderline intermittent $\mathrm{EH}$ and in normotensive offspring of hypertensive parents, suggesting a familial predisposition in which CR represents a primary vascular abnormality that antedates the onset of sustained elevation of blood pressure (BP) $[6,7]$. We recently reported the unexpected finding that LBW infants born at term or preterm to normotensive mothers do not have CR: instead these infants have a significantly higher dermal $\mathrm{CD}$ at birth compared to their normal birth weight (NBW) counterparts [8]. It is becoming increasingly evident that conditions early in life can influence adult diseases. It has been reported in some studies that hyperoxia during the neonatal period may have a negative effect on the cardiovascular system [9]. We hypothesized that the high 
capillary density seen in the LBW infants at birth was an inutero compensatory adaptation to unfavorable conditions and that after birth, with the availability of adequate nutrition and more importantly oxygen, an accelerated capillary remodeling then ensues $[10,11]$. We set out to test our hypothesis by conducting serial measurements of skin capillary density in LBW preterm infants receiving oxygen therapy.

\section{Methods}

\section{Study subjects}

The study was approved by the London - Riverside Research Ethics Committee (13/LO/1449) and was conducted at St. George's University Hospitals NHS Foundation Trust, London, UK. Written informed consent was obtained from all parents. The infants in the study were recruited from the neonatal intensive care unit (NICU), Transitional Care Unit and the Postnatal Ward. We studied $26 \mathrm{LBW}$ infants $(<2500 \mathrm{~g}$ and $\geq 30$ weeks of gestation) and $14 \mathrm{NBW}$ infants as controls. As this was a pilot pragmatic study, no official power calculation was done to determine sample size. We excluded infants with sepsis, chromosomal or congenital abnormalities and those requiring surgery. The antenatal history of the mothers and details of the findings during the anomaly ultrasound scan were also recorded. Dietary habits, smoking history, family history of diabetes, ischemic heart disease, stroke, hypercholesterolemia, and hypertension were obtained from both parents.

\section{Handheld Video Capillaroscopy System (HVCS)}

\section{CapiScope Handheld Video Capillaroscopy System} (HVCS, KK technology, Devon, England) was used to measure skin capillary density at the plantar surface of the infant big toe according to a previously well-validated protocol $[8,12,13]$. The size of the microscopic field was $0.81 \mathrm{~mm}^{2}$, with an image size of $1280 \times 1024$ pixels and a field view of $1037 \times 829$ pixels. The optical illumination of the HVCS device was done using four $525 \mathrm{~nm}$ light sources. The live images were recorded onto a Panasonic DMREX99VEBK HDD recorder. Disposable sterile probe covers were used for imaging to minimize the risk of infection. Four microscopic fields, $0.81 \mathrm{~mm}^{2}$ each, were recorded continuously for $30 \mathrm{~s}$. The number of all capillaries (i.e., with stagnant, intermittently flowing and continuously flowing red blood cells) was counted. Basal capillary density (BCD), which represents functional capillary density, was calculated as the mean of these four microscopic fields. We used venous congestion to maximize the number of visualized perfused skin capillaries by applying a neonatal
BP cuff (Heine Gamma 7 Sphygmomanometer, Germany) around the calf muscle. The cuff was then inflated and maintained at $30 \mathrm{~mm} \mathrm{Hg}$ for $2 \mathrm{~min}$, and further images were recorded continuously for $2 \mathrm{~min}$ to determine the maximal capillary density (MCD), which represents structural capillary density. Capillaries were counted off line using the CapiScope computer software (KK-Technology, Exeter, UK). Skin temperature was monitored during the study using an YSI Tele-thermometer (YSI Inc., Dayton, $\mathrm{OH}, \mathrm{USA}$ ).

In LBW infants not receiving oxygen therapy, capillaroscopy was performed immediately after birth (baseline measurement) provided the cardiorespiratory status was stable and repeat follow-up measurements were done after 5 days and 10 days. The baseline measurement in LBW infants requiring oxygen treatment was slightly delayed due to the medical condition of the infants. In preterm LBW infants an additional measurement of capillary density was taken at the corrected (i.e. 40 weeks) term age prior to their discharge from hospital or by visiting them at home. In the NBW group we measured capillary density at birth only before mothers were discharged from the hospital. Blood and urine samples were obtained from the infants.

\section{BP measurement}

We used the Welch Allyn VSM $300^{\mathrm{TM}}$ series monitor to measure BP in infants at each visit while they were sleeping or feeding to avoid movement artefacts. An appropriate sized disposable Welch Allyn neonatal cuff (size, 1-4) was used to measure the BP. All measurements were taken in the lower limb, as this was the easily accessible part to the neonate inside the incubator.

\section{Proteome array}

Blood samples were obtained from 13 LBW infants during their stay in the ICU by venepuncture or heel prick method. The samples were allowed to clot for $30 \mathrm{~min}$ and after centrifugation the serum was aliquoted and stored at $-80^{\circ}$ C. Two hundred and one cytokines were measured by the Quantibody Human Cytokine Antibody Array 4000 (RayBiotech, Norcross, GA, USA) according to manufacturer's instructions.

\section{Statistical analysis}

The primary endpoint was the change in maximal (structural) skin capillary density during venous congestion (MCD) between birth to adjusted age of 40 weeks postnatal life. Shapiro-Wilk test was used to assess the normality of study parameters. ANOVA test with Bonferroni correction was used for comparison of means among the groups. 
Student's $t$ test was used to compare the difference of capillary density measurement from baseline to the corrected term age in the preterm LBW infants. Chi-square test was used to compare proportions between the LBW and NBW mothers. Pearson correlation coefficient was used to describe the linear correlation between capillary density and birth weight and changes in BP. Statistical significance was declared when the $p$-value was $<0.05$. All statistical analysis was carried out using the IBM SPSS 22 (IBM Corporation, Armonk, NY, USA).

\section{Results}

Table 1 shows the baseline characteristics of study subjects. There were no significant differences in maternal age, body mass index, booking BP (i.e. BP measured during the first antenatal clinic visit), pulsatility index, or ultrasound fetal parameters during the anomaly scan between the groups. Of the 26 LBW infants we studied, 10 received oxygen treatment either by continuous positive airway pressure, mechanical ventilation, or a nasal cannula.

At birth, LBW infants had a significantly higher BCD (mean difference +9.3 cap/field, $95 \%$ CI: $1.5-17.1, p=$ 0.021 ) and MCD (mean difference +11.6 cap/field, $95 \%$ CI: $1.6-21.7, p=0.025)$ compared to the NBW infants. Compared to their NBW counterparts, LBW infants had a significantly lower diastolic BP (mean difference -10.1 $\mathrm{mm} \mathrm{Hg}, 95 \% \mathrm{CI}:-17.7$ to $-2.6, p=0.010$ ) (Fig. 1). There was no significant difference in systolic BP. Pulse rate was significantly higher in LBW (mean difference $15.1 \pm 10.1$ beats/min, 95\% CI: 3.4-26.8, $p=0.013$ ). There was a significant correlation between birth weight and both BCD ( $\mathrm{r}$ $=-0.309, p=0.052)$, and MCD in the entire group $(r=$ $-0.355, p=0.025$ ).

At adjusted age of 40 weeks, the LBW oxygen group showed a significant reduction in $\mathrm{BCD}$ (mean difference -20.4 cap/field, 95\% CI: -7.2 to $-33.6, p=0.009$ ) and MCD (mean difference -20.6 cap/field, $95 \% \mathrm{CI}$ : -3.7 to $-37.5, p=0.025)$ compared to baseline values at birth. Similarly the LBW nonoxygen group had a significant reduction in $\mathrm{BCD}$ (mean difference -26.7 cap/field, 95\% CI -16.3 to $-37.1, p<0.0001$ ) and MCD (mean difference -28.2 cap/field, $95 \% \mathrm{CI},-17.1$ to $-39.2, p<0.001)$. There were no significant differences in BCD and MCD between the two LBW groups. The reduction in $\mathrm{CD}$ was associated with a significant rise in systolic $(r=-0.385, p<0.035)$, and diastolic BP $(r=-0.361, p<0.050)$ in both LBW groups. The rise in systolic BP and diastolic BP was more pronounced in the LBW oxygen group (mean difference $24 \mathrm{~mm}$ $\mathrm{Hg}, 95 \% \mathrm{CI}: 4-11, p=0.004$ for systolic BP, and mean difference $11 \mathrm{~mm} \mathrm{Hg}$, 95\%CI: 3-19, $p=0.014$ for diastolic $\mathrm{BP}$ ) compared to the LBW nonoxygen group (mean difference $16 \mathrm{~mm} \mathrm{Hg}, 95 \% \mathrm{CI}: 4-6, p=0.005$ and mean difference $13 \mathrm{~mm} \mathrm{Hg}, 95 \% \mathrm{CI}: 3-4, p=0.01$, respectively) (Table 2).

We found no significant differences in angiogenic or antiangiogenic factors between the two LBW groups. Macrophage colony-stimulating factor was significantly lower in the LBW oxygen group compared to the LBW nonoxygen group $(0.656 \pm 0.389$ versus $0.217 \pm 0.195 \mathrm{pg} /$ $\mathrm{ml}, p<0.035$ ), but we are unable to explain the significance of this finding.

\section{Discussion}

The study demonstrates that premature LBW infants who received oxygen therapy developed significant functional and structural CR, which was associated with a significant increase in both systolic and diastolic BPs at adjusted 40 weeks of age. We also found that LBW infants who did not receive oxygen therapy developed similar $\mathrm{CR}$, but the increase in their BP was not as significant as in those who received oxygen therapy. In addition, we confirmed our previous report that LBW infants have higher functional and structural capillary densities at birth compared to NBW infants [8]. The effect of preterm birth on microvascular development has also been highlighted by studies showing reduced retinal vascular caliber and density (independently of retinopathy of prematurity), as well as reduced cutaneous capillary density in children and young adults born very preterm [14, 15]. As previously stated, it has become increasingly evident that conditions early in life can influence adult diseases, but the underlying mechanisms are unkown [16]. Recent data suggest that perinatal oxidative stress may be one of the initiating triggers in long-term programming of cardiovascular function. Our results are in agreement with several preclinical studies. In animal models, the continuous supplementation of oxygen has been shown to affect the development of microvasculature and to induce vascular obliteration and CR [17, 18] and an increase in BP [19]. Yzydorczyk et al. [9] studied Sprague-Dawley pups who were exposed to $80 \%$ oxygen from 3-10 days after birth and found that in both male and female rats exposed to oxygen as newborns, systolic, and diastolic BP were increased by about $15 \mathrm{~mm} \mathrm{Hg}$, capillary density was reduced by $30 \%$ and the number of nephrons per kidney was decreased by $25 \%$. They suggested that neonatal hyperoxia leads in the adult rat to increased BP, vascular dysfunction, CR, and reduced nephron number. It has been shown that the nephron numbers are decreased in adult individuals with EH [20] and in intrauterine growth-restricted infants [21, 22].

Milstein et al. [19] studied sublingual microvascular vessel density, vessel diameters, and microvascular flow in rabbits breathing sequential oxygen/air mixtures under 
Table 1 Baseline characteristics of study subjects

\begin{tabular}{|c|c|c|c|c|}
\hline Variables & $\begin{array}{l}\text { LBW oxygen } \\
(n=10)\end{array}$ & $\begin{array}{l}\text { LBW non- } \\
\text { oxygen }(n=16)\end{array}$ & $\begin{array}{l}\text { NBW control } \\
(n=14)\end{array}$ & $P$ - value ANOVA \\
\hline \multicolumn{5}{|l|}{ Maternal data } \\
\hline Age (years) & $34 \pm 2.0$ & $34 \pm 7.8$ & $31 \pm 4.0$ & 0.198 \\
\hline BMI $\left(\mathrm{kg} / \mathrm{m}^{2}\right)$ & $27.0 \pm 6.8$ & $30.2 \pm 6.3$ & $28.2 \pm 5.4$ & 0.447 \\
\hline \multicolumn{5}{|l|}{ Blood pressure (mm Hg) } \\
\hline Systolic & $123 \pm 14$ & $125 \pm 13$ & $114 \pm 17$ & 0.161 \\
\hline Diastolic & $77 \pm 14$ & $77 \pm 16$ & $72 \pm 7$ & 0.614 \\
\hline \multicolumn{5}{|l|}{ Ethnicity $n(\%)$} \\
\hline Caucasian & $4(40)$ & $9(56.3)$ & $7(50)$ & 1.0 \\
\hline South Asian & $2(20)$ & $4(25)$ & $5(35.8)$ & 1.0 \\
\hline Afro Caribbean & $2(20)$ & $3(18.7)$ & $1(7.1)$ & 1.0 \\
\hline Mixed & $2(20)$ & 0 & $1(7.1)$ & 1.0 \\
\hline HDP & $5(50)$ & $6(37.5)$ & $1(7.1)$ & 0.059 \\
\hline Family history of HTN & $3(30)$ & $8(50)$ & $10(71.4)$ & 0.324 \\
\hline \multicolumn{5}{|l|}{ Prenatal data } \\
\hline Bi-Parietal diameter $(\mathrm{cm})$ & $54.2 \pm 3.8$ & $52.3 \pm 2.4$ & $53.2 \pm 2.4$ & 0.258 \\
\hline Head circumference $(\mathrm{cm})$ & $182.5 \pm 25.9$ & $186.8 \pm 7.3$ & $178.8 \pm 43.2$ & 0.780 \\
\hline Femur length $(\mathrm{cm})$ & $34.7 \pm 3.3$ & $35.3 \pm 1.4$ & $36.2 \pm 1.5$ & 0.227 \\
\hline Abdominal Circumference (cm) & $161.5 \pm 14.2$ & $162.4 \pm 8.6$ & $167.2 \pm 11.0$ & 0.409 \\
\hline Uterine artery PI & $2.79 \pm 0.89$ & $2.53 \pm 0.74$ & $2.42 \pm 0.56$ & 0.640 \\
\hline \multicolumn{5}{|l|}{ Neonatal data } \\
\hline Birth weight (g) & $1645 \pm 418$ & $1715 \pm 377$ & $3388 \pm 558$ & 0.0001 \\
\hline Gestational age (weeks) & $32.5 \pm 1.4$ & $33.3 \pm 1.7$ & $39.1 \pm 2.29$ & 0.0001 \\
\hline Age at capillaroscopy (days) & $9 \pm 6$ & $4 \pm 4$ & $2 \pm 1$ & 0.001 \\
\hline Gestational age at analysis & $33.8 \pm 1.7$ & $34.0 \pm 1.6$ & $39.3 \pm 2.1$ & 0.0001 \\
\hline Weight on study day (g) & $1513 \pm 299$ & $1740 \pm 331$ & $3388 \pm 588$ & 0.0001 \\
\hline \multicolumn{5}{|l|}{ Capillary density (per $\mathrm{mm}^{2}$ ) } \\
\hline Basal & $92 \pm 15$ & $93 \pm 18$ & $83 \pm 8$ & 0.155 \\
\hline Maximal & $97 \pm 17$ & $101 \pm 17$ & $87 \pm 11$ & 0.069 \\
\hline Skin temperature $\left({ }^{\circ} \mathrm{C}\right)$ & $34.8 \pm 1.5$ & $34.9 \pm 1.2$ & $33.7 \pm 1.2$ & 0.106 \\
\hline Room temperature $\left({ }^{\circ} \mathrm{C}\right)$ & $24.6 \pm 0.97$ & $25.2 \pm 1.5$ & $26.7 \pm 1.2$ & 0.001 \\
\hline \multicolumn{5}{|l|}{ Blood pressure (mm Hg) } \\
\hline Systolic & $61 \pm 9$ & $68 \pm 13$ & $70 \pm 11$ & 0.168 \\
\hline Diastolic & $33 \pm 6$ & $38 \pm 10$ & $45 \pm 12$ & 0.022 \\
\hline Mean & $43 \pm 8$ & $48 \pm 10$ & $53 \pm 11$ & 0.104 \\
\hline Heart rate & $145 \pm 15$ & $131 \pm 17$ & $122 \pm 14$ & 0.007 \\
\hline \multicolumn{5}{|l|}{ Laboratory results } \\
\hline Hemoglobin & $147 \pm 20$ & $180 \pm 26$ & $171 \pm 21$ & 0.022 \\
\hline Hematocrit & $46 \pm 6.3$ & $55 \pm 8.4$ & $56 \pm 0.1$ & 0.035 \\
\hline Bilirubin & $143 \pm 70$ & $156 \pm 40$ & $123 \pm 1.4$ & 0.803 \\
\hline $\mathrm{pO}_{2}$ & $6.7 \pm 1.5$ & $6.4 \pm 1.5$ & NA & 0.827 \\
\hline $\mathrm{pCO}_{2}$ & $6.3 \pm 0.9$ & $5.9 \pm 0.8$ & NA & 0.166 \\
\hline Duration of $\mathrm{O}_{2}$ exposure (h) & $347.8 \pm 524.8$ & & & \\
\hline Maximum $\mathrm{FiO}_{2}$ given & $59.6 \pm 34.2$ & & & \\
\hline
\end{tabular}

$L B W$ low birth weight, $N B W$ normal birth weight, $\mathrm{HDP}$ hypertensive disorder of pregnancy, $\mathrm{FiO}_{2}$ fractional percentage of inspired oxygen 

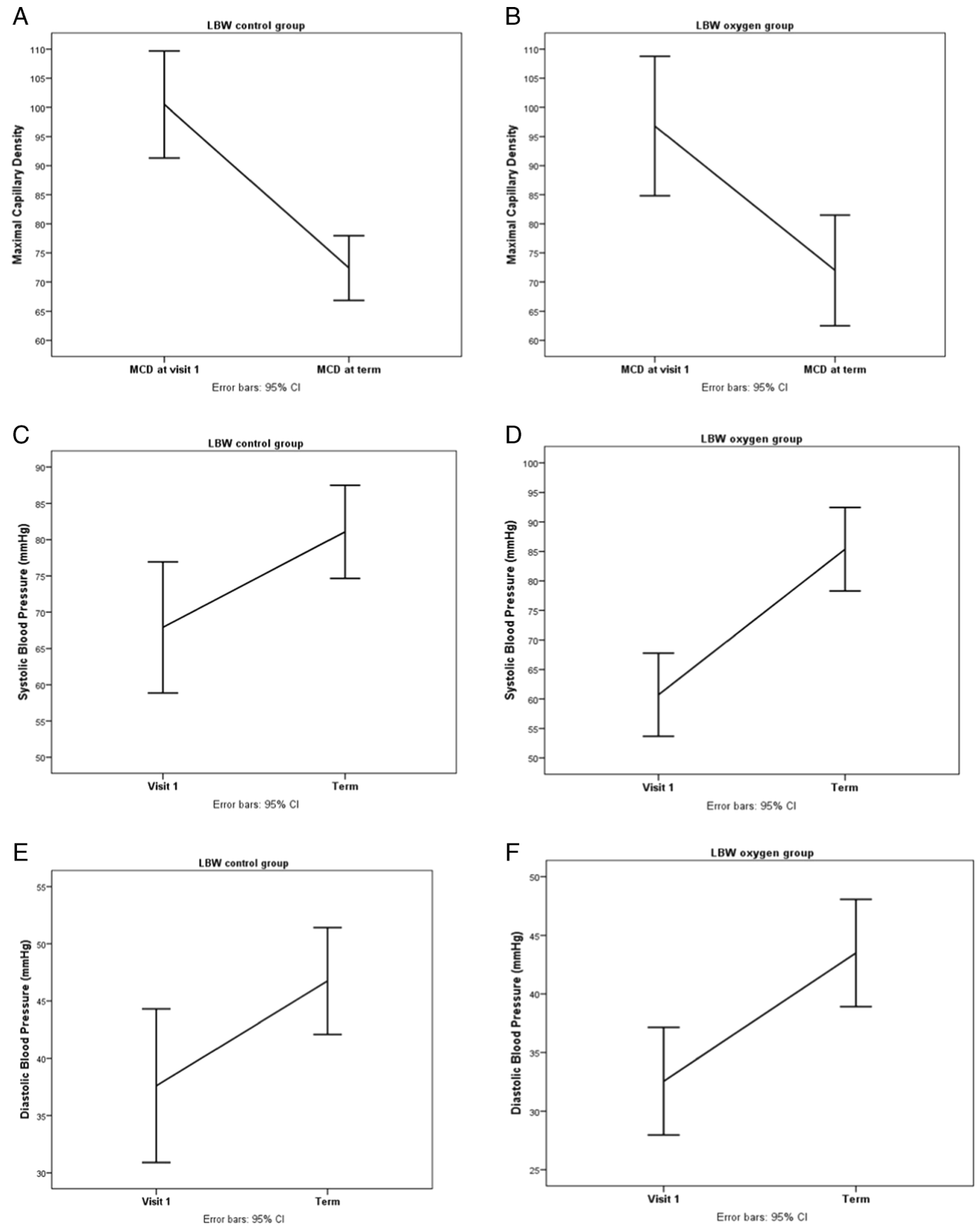

Fig. 1 Changes in the capillary density $(\mathbf{a}, \mathbf{b})$, systolic blood pressure $(\mathbf{c}, \mathbf{d})$ and diastolic blood pressure (e, f) from baseline measurement to adjusted 40 weeks of age in preterm LBW infants. Both the LBW

oxygen group and the LBW control group showed a statistically significant reduction in the capillary density $(\mathbf{a}, \mathbf{b})$ followed by increase in the blood pressure $(\mathbf{c}-\mathbf{f})$ 
Table 2 Comparison between LBW babies at adjusted 40th week who were treated or not treated with oxygen compared to NBW infants at term

\begin{tabular}{|c|c|c|c|c|}
\hline Variables & $\begin{array}{l}\text { LBW oxygen } \\
(n=10) \text { (40th week) }\end{array}$ & $\begin{array}{l}\text { LBW nonoxygen } \\
(n=16) \text { (40th week) }\end{array}$ & $\begin{array}{l}\text { NBW control } \\
(n=14) \text { (Term age) }\end{array}$ & $P$-value ANOVA \\
\hline \multicolumn{5}{|c|}{ Capillary density (per $\mathrm{mm}^{2}$ ) } \\
\hline Basal & $69 \pm 12$ & $66 \pm 11$ & $83 \pm 8$ & 0.0001 \\
\hline Maximal & $72 \pm 11$ & $72 \pm 9$ & $87 \pm 11$ & 0.005 \\
\hline \multicolumn{5}{|c|}{ Blood pressure $(\mathrm{mm} \mathrm{Hg})$} \\
\hline Systolic & $85 \pm 9$ & $81 \pm 10$ & $70 \pm 11$ & 0.006 \\
\hline Diastolic & $49 \pm 7$ & $47 \pm 8$ & $45 \pm 12$ & 0.742 \\
\hline Heart rate & $160 \pm 11$ & $158 \pm 14$ & $122 \pm 14$ & 0.0001 \\
\hline
\end{tabular}

$L B W$ low birth weight, $N B W$ normal birth weight normobaric and hyperbaric conditions. They found that normobaric hyperoxia produced significant microvascular rarefaction and significant increases in systolic and mean BP when compared to normobaric normoxia. Of interest they found that all microcirculatory abnormalities reverted back to normal values upon return to normoxia.

Ashton et al. [23] confirmed that $80 \%$ inspired oxygen in healthy kittens caused "vaso-obliteration" of the newly formed capillaries; when the animals were returned to ambient air, a "vaso-proliferative" effect was observed causing retinopathy of prematurity. Oxygen supplementation in humans during the neonatal period has proven adverse effects on the microvascular circulation especially in the retina and the lungs. However it has been shown previously that capillary density ordinarily decreases after birth in preterm infants who did not receive oxygen therapy. Kroth et al. measured basal or functional small vessel density (FSVD) in 25 preterm infants born $<30$ weeks old using orthogonal polarization spectral imaging on their upper arm. They found that FSVD decreased at 4 weeks compared to week 1 . However, they did not observe any significant change in BP, but observed a negative correlation between FSVD and systolic BP [24]. Similarly van Elteren et al. [25] measured total vascular density (TVD) using incident dark field technology in 60 preterm infants born less than 32 weeks and 33 term infants during the first month of life. Similar to us, they found that TVD was higher in preterm infants at birth and that there was a progressive decline in TVD from birth to 28 days in preterm infants.

Our results corroborate with Kistner et al. who found that preterm-born women had significant rarefaction of retinal vessels manifested as fewer numbers of vascular branching points compared with NBW controls. This was associated with an increased casual BP suggesting that being born preterm does have effects on the vascular system that persist into adult life [15].

It has been reported that premature infants have decreased antioxidant defenses and are exposed upon birth to high oxygen concentration relative to the intrauterine environment [26]. Additional oxygen therapy may therefore cause oxidative tissue damage, leading to pathologies such as retinopathy of prematurity and broncho-pulmonary dysplasia [27]. More recent studies indicate that individuals with a history of premature birth exhibit higher BP levels and abnormal retinal microvasculature and parameters of cardiovascular dysfunction [15, 28]. While the mechanisms linking prematurity to adult cardiovascular disorders are unknown, our data and that from others support a putative role for neonatal oxidative stress. Oxidative stress has been shown to be involved in the promotion of rarefaction through endothelial apoptosis in hypertensive rats, while treatment with antioxidants has resulted in a reduction of microvessel loss [29].

We acknowledge the limitations in our study that include the small numbers of LBW infants treated with oxygen, but this highlights the difficulties in recruiting such infants while they are in the NICU. The ethnicity of infants was not even in study groups and the significant difference in the age of infants on the study day may have been a confounding factor [30]. However, we assessed BCD and MCD in NBW term infants of different ethnic backgrounds born to normotensive mothers and found no difference in capillary density between the different groups (unpublished data). There was also significant variability in the mode of oxygen therapy, duration and percentage of fractional percentage of inspired oxygen $\left(\mathrm{FiO}_{2}\right)$ received in the postnatal period. It was not possible to control for the above factors, or render them uniform for the study, as they were tailored according to the clinical needs of each preterm infant. The timing of baseline measurement in the LBW oxygen group was slightly delayed compared to the nonoxygen group because of limited access to the NICU due to the medical condition of the baby. However, the mean gestational age was closely matched during the serial measurement of capillary density between the groups. All the blood samples were taken within the first 2 weeks in the NICU as we aimed for the blood sampling to coincide with routine clinical blood sampling to avoid additional discomfort to the baby. Similarly, BP measurements were taken only once, again to avoid undue discomfort to the infants. We have previously observed that sequential BP measurements 
awaken infants and disturb them, such that second and third readings were almost always higher than the first reading. We therefore ensured that all the BP measurements were taken while the baby was sleeping or feeding to minimize any artefacts.

In conclusion, oxygen therapy in premature LBW infants in the neonatal period is associated with higher systolic and diastolic BP levels, but has no significant effect on the rate of capillary reduction from birth to adjusted age of 40 weeks. Further studies are needed to investigate the humoral factors that trigger the microcirculatory changes in LBW infants during the neonatal period, which will be of importance in preventing future hypertension and cardiovascular diseases in later life.

\section{Summary table}

\section{"What is known about topic"}

- LBW and premature birth are known risk factors for future cardiovascular disease and in particular EH.

- $\mathrm{CR}$ is an established hallmark of EH and is known to occur in individuals with a history of LBW.

- It has been shown recently that LBW infants do not have $\mathrm{CR}$ at birth, but rather increased capillary density compared to NBW.

- In preclinical animal studies, oxygen therapy has been shown to induce vascular obliteration and CR in the new born.

\section{"What this study adds"}

- Oxygen therapy in premature LBW infants in the neonatal period is associated with higher systolic and diastolic BP levels but has no significant effect on the rate of capillary reduction.

- Further studies are needed to investigate the humoral factors that trigger the microcirculatory changes in LBW infants during the neonatal period, which will be of importance in preventing future hypertension and cardiovascular diseases in later life.

Funding St George's Charitable Foundation Medical Research Grant and Molecular \& Clinical Sciences Research Institute.

\section{Compliance with ethical standards}

Conflict of interest The authors declare that they have no conflict of interest.

Publisher's note: Springer Nature remains neutral with regard to jurisdictional claims in published maps and institutional affiliations.

\section{References}

1. Barker DJ, Winter PD, Osmond C, Margetts B, Simmonds SJ. Weight in infancy and death from ischaemic heart disease. Lancet. 1989;2:577-80.

2. Curhan GC, Willett WC, Rimm EB, Spiegelman D, Ascherio AL, Stampfer MJ. Birth weight and adult hypertension, diabetes mellitus, and obesity in US men. Circulation. 1996;94:3246-50.

3. Levy BI, Schiffrin EL, Mourad JJ, Agostini D, Vicaut E, Safar $\mathrm{ME}$, et al. Impaired tissue perfusion: a pathology common to hypertension, obesity, and diabetes mellitus. Circulation. 2008;118:968-76.

4. Martin H, Hu J, Gennser G, Norman M. Impaired endothelial function and increased carotid stiffness in 9-year-old children with low birthweight. Circulation. 2000;102:2739-44.

5. Antonios TFT, Singer DR, Markandu ND, Mortimer PS, MacGregor GA. Structural skin capillary rarefaction in essential hypertension. Hypertension. 1999;33:998-1001.

6. Antonios TF, Rattray FM, Singer DR, Markandu ND, Mortimer PS, MacGregor GA. Rarefaction of skin capillaries in normotensive offspring of individuals with essential hypertension. Heart. 2003;89:175-8.

7. Antonios TFT, Singer DR, Markandu ND, Mortimer PS, MacGregor GA. Rarefaction of skin capillaries in borderline essential hypertension suggests an early structural abnormality. Hypertension. 1999;34:655-8.

8. D'Souza R, Raghuraman RP, Nathan P, Manyonda IT, Antonios TF. Low birth weight infants do not have capillary rarefaction at birth: implications for early life influence on microcirculation. Hypertension. 2011;58:847-51.

9. Yzydorczyk C, Comte B, Cambonie G, Lavoie JC, Germain N, Ting Shun Y, et al. Neonatal oxygen exposure in rats leads to cardiovascular and renal alterations in adulthood. Hypertension. 2008;52:889-95.

10. Struijker-Boudier HA, Heijnen BF. Early life microcirculation and the development of hypertension. Hypertension. 2011;58:768-9.

11. Struijker-Boudier HA, Heijnen BF, Liu YP, Staessen JA. Phenotyping the microcirculation. Hypertension. 2012;60:523-7.

12. Raghuraman RP, D'Souza R, Nathan P, Wang D, Manyonda IT, Antonios TF. Skin capillary density in infants born to normotensive mothers: a comparison between singleton and twin infants. Microcirculation. 2014;21:67-73.

13. Antonios TF, Raghuraman RP, D'Souza R, Nathan P, Wang D, Manyonda IT. Capillary remodeling in infants born to hypertensive pregnancy: pilot study. Am J Hypertens. 2012;25:848-53.

14. Bonamy AK, Martin H, Jorneskog G, Norman M. Lower skin capillary density, normal endothelial function and higher blood pressure in children born preterm. J Intern Med. 2007;262:635-42.

15. Kistner A, Jacobson L, Jacobson SH, Svensson E, Hellstrom A. Low gestational age associated with abnormal retinal vascularization and increased blood pressure in adult women. Pedia Res. 2002;51:675-80.

16. Nuyt AM. Mechanisms underlying developmental programming of elevated blood pressure and vascular dysfunction: evidence from human studies and experimental animal models. Clin Sci. 2008;114:1-17.

17. Penn JS, Henry MM, Tolman BL. The range of oxygen variation determines the degree of retinopathy in newborn rats exposed to variable atmospheric oxygen. Investig Ophthalmol Vis Sci. 1994;35:1653-1653.

18. Benderro GF, Sun X, Kuang Y, Lamanna JC. Decreased VEGF expression and microvascular density, but increased HIF-1 and 2alpha accumulation and EPO expression in chronic moderate hyperoxia in the mouse brain. Brain Res. 2012;1471:46-55. 
19. Milstein DM, Helmers R, Hackmann S, Belterman CN, van Hulst RA, de Lange J. Sublingual microvascular perfusion is altered during normobaric and hyperbaric hyperoxia. Micro Res. 2016;105:93-102.

20. Keller G, Zimmer G, Mall G, Ritz E, Amann K. Nephron number in patients with primary hypertension. N Engl J Med. 2003;348:101-8.

21. Manalich R, Reyes L, Herrera M, Melendi C, Fundora I. Relationship between weight at birth and the number and size of renal glomeruli in humans: a histomorphometric study. Kidney Int. 2000;58:770-3.

22. Hughson M, Farris AB 3rd, Douglas-Denton R, Hoy WE, Bertram JF. Glomerular number and size in autopsy kidneys: the relationship to birth weight. Kidney Int. 2003;63:2113-22.

23. Ashton N, Ward B, Serpell G. Effect of oxygen on developing retinal vessels with particular reference to the problem of retrolental fibroplasia. Br J Ophthalmol. 1954;38:397-432.

24. Kroth J, Weidlich K, Hiedl S, Nussbaum C, Christ F, Genzelboroviczeny O. Functional vessel density in the first month of life in preterm neonates. Pediatr Res. 2008;64:567-71.
25. van Elteren HA, de Jonge RC, van Rosmalen J, Ince C, Reiss IK. Adaptation of the cutaneous microcirculation in preterm neonates. Microcirculation. 2016;23:468-74.

26. Thibeault DW. The precarious antioxidant defenses of the preterm infant. Am J Perinatol. 2000;17:167-81.

27. Hardy P, Dumont I, Bhattacharya M, Hou X, Lachapelle P, Varma $\mathrm{DR}$, et al. Oxidants, nitric oxide and prostanoids in the developing ocular vasculature: a basis for ischemic retinopathy. Cardiovasc Res. 2000;47:489-509.

28. Siewert-Delle A, Ljungman S. The impact of birth weight and gestational age on blood pressure in adult life: a population-based study of 49-year-old men. Am J Hypertens. 1998;11:946-53.

29. Kobayashi N, DeLano FA, Schmid-Schonbein GW. Oxidative stress promotes endothelial cell apoptosis and loss of microvessels in the spontaneously hypertensive rats. Arterioscler Thromb Vasc Biol. 2005;25:2114-21.

30. Nama V, Onwude J, Manyonda IT, Antonios TF. Is capillary rarefaction an independent risk marker for cardiovascular disease in South Asians? J Hum Hypertens. 2011;25:465-6. 